\title{
Editorial: The Role of Platelets in Cancer Progression and Malignancy
}

\author{
Martin Schlesinger ${ }^{1}$, Martina Gobec ${ }^{2}$ and Alexander T. Bauer ${ }^{3 *}$ \\ 1 Pharmaceutical Institute, University of Bonn, Bonn, Germany, 2 Faculty of Pharmacy, University of Ljubljana, Ljubljana, \\ Slovenia, ${ }^{3}$ Department of Dermatology and Venereology, University Hospital Hamburg-Eppendorf, Hamburg, Germany
}

Keywords: platelets, cancer, metastasis, thrombosis, inflammation, biomarkers, therapeutic targets

Editorial on the Research Topic

The Role of Platelets in Cancer Progression and Malignancy

The association between malignancy and coagulation is known since more than 150 years and was initially described by Armand Trousseau (1). Thrombotic complications are not only a diagnostic hind for an occult cancer (2), venous thromboembolism is also the second leading cause of death in cancer patients (3). Although novel treatment strategies, such as immune checkpoint inhibition or CAR T cell therapy have revolutionized cancer therapy and have drastically improved the prognosis for some tumor entities, a high incidence of thrombotic events is recognized $(4,5)$. The same studies clearly identify a correlation between thrombosis, treatment side effects and an increased mortality. In this context, platelets are known for their crucial roles linking coagulation, metastasis and the local immune regulation (6). For instance, platelets secrete proinflammatory cytokines and adhere to leukocytes (7). When interacting with $\mathrm{T}$ cells, platelets can selectively modulate $\mathrm{T}$ cell activation and recruitment (8), and are also able to impact the function of further leukocyte populations (9). However, although best recognized for their roles in hemostasis and inflammation, the underlying mechanisms of this complex interplay in malignancy are poorly understood.

In this Research Topic on the role of platelets in cancer progression and malignancy, we compiled three reviews and two research articles. The reviews address the impact of platelets on liver disease and cancer, platelet-tumor cell interactions in the different steps of the metastatic cascade and the underlying molecular mechanisms. Two original research articles evaluate the role of platelets as potential biomarkers for breast cancer progression and the impact of platelets in thrombosis and tumor growth in pancreatic tumors.

In the past, several reports underline the relevance of platelets for the pathophysiology of the liver (10). Marie et al. present findings on the crucial role of platelets in the course of liver disease. The current knowledge of how liver injury results in a dysregulated coagulation and alters platelet function is discussed. Vice versa, it was recently shown that platelets contribute to the conversion of non-alcoholic steatohepatitis to liver cirrhosis and ultimately hepatocellular carcinoma (11). Platelet adhesion, platelet activation and granule secretion were identified as pivotal for hepatocarcinogenesis. Significantly, analogous mechanisms are involved in the development of metastasis. A special focus on the impact of platelets in the different steps of metastasis formation and how circulating platelets orchestrate cancer cell invasion, intravasation, the survival of tumor cells in the circulation and extravasation is given by the mini review of Fabricius et al. Particularly, platelet-derived P-selectin is highlighted and preclinical 
evidence demonstrated that the binding of platelets via P-selectin not only protects circulating tumor cells in the blood stream but also promotes tumor cell extravasation. However, a critical view on the experimental data imply fundamental species-specific differences between murine and human platelets in P-selectin levels and binding to their ligands on cancer cells. This could be a plausible explanation for the largely unrealized potential of anti-platelet therapies in malignancy and many mechanistic questions remain to be answered to ensure future clinical applications. With similar motivation in mind, Braun et al., offer an extended literary overview of the molecular mechanisms of tumor cell-platelet interactions. This review article advances our understanding about the role of platelets in cancer by presenting relevant contributions of platelets to cancer-associated thrombosis and cancer-associated inflammation. Circulating platelets are equipped with a vast amount of molecules involved in metastasis and thrombosis, e. g. VEGF-A (12), P-selectin (13), VWF (14) and platelet factor 4 (15). Consequently, the identification of molecules associated with activated platelets represent promising candidates to serve as predictive and prognostic clinical biomarkers. The group of Hinterleitner et al. addresses the potential use of the plateletderived Tumor necrosis factor Receptor TNFRSF13B (TACl) as biomarker in breast cancer patients. Using immunofluorescent analysis and flow cytometry the authors provide clear evidence of an increased TACl expression on the surface of platelets isolated of breast cancer patients when compared to healthy donors. Interestingly, platelet $\mathrm{TACl}$ inversely correlated to the disease stages. Patients with metastases exhibited less platelet expression than patients with a localized primary tumor. Thus, TACl on platelets may have great clinical potential in predicting breast cancer and seems to provide important predictive value for the occurrence of metastasis. Based on the critical role of platelets in tumor progression, and the existence of abundant literature on antiplatelet strategies in malignancy, one may speculate that antiplatelet therapies could be promising for cancer patients (16). Although preclinical data show interesting results using inhibitors for purinergic ADP receptors on the platelet surface (17), the results from clinical trials are conflicting. Treatment with clopridrogel even increased the incidence for the development of cancer and the risk of tumor-related mortality $(16,18)$. This highlights the urgent need for further mechanistic studies for a better understanding of molecular mechanisms of platelet inhibition in the context of cancer and thrombosis. Finally, this intention has been taken up by the experimental study of Palacios-Acedo et al. Using a combination of in vitro systems and a mouse model for pancreatic cancers, the authors make the important observation that compared to aspirin, clopidrogel exhibited a superior therapeutic benefit by inhibition of the proliferation of pancreatic adenocarcinoma cells. Importantly, work in preclinical models for malignant melanoma have shown that platelets suppress $\mathrm{T}$ cell functions and emphasize the validity of aspririn and clopidrogel for combinations with immunotherapeutics (19).

Overall, the collection of articles in this Research Topic highlights platelets as important link between thrombosis, treatment side effects and tumor progression. Hence, a deepening of our understanding of cancer-related coagulation

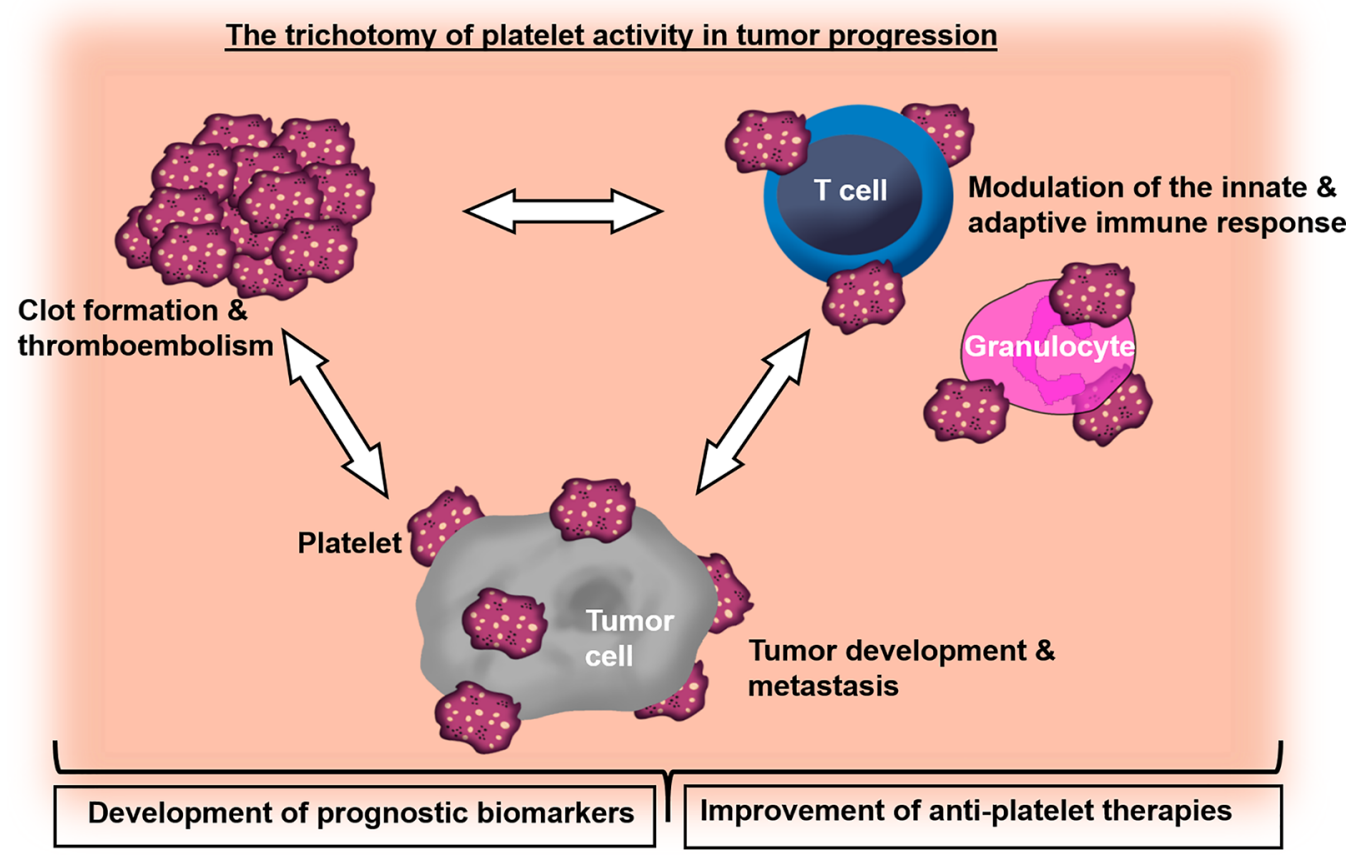

FIGURE 1 | Graphical overview demonstrating the role of platelets in the crosstalk of cancer-associated thrombosis, inflammation and tumor progression. 
and inflammation with regard to platelet functions will accelerate the design of novel treatment strategies to target thrombosis and metastasis in the future (Figure 1).

\section{AUTHOR CONTRIBUTIONS}

All authors listed have provided a substantial, direct and intellectual contribution to the manuscript and have approved it before publication.

\section{REFERENCES}

1. Varki A. Trousseau's Syndrome: Multiple Definitions and Multiple Mechanisms. Blood (2007) 110:1723-9. doi: 10.1182/blood-2006-10-053736

2. Monreal M, Lensing AW, Prins MH, Bonet M, Fernandez-Llamazares J, Muchart J, et al. Screening for Occult Cancer in Patients With Acute Deep Vein Thrombosis or Pulmonary Embolism. J Thromb Haemost (2004) 2:87681. doi: 10.1111/j.1538-7836.2004.00721.x

3. Khorana AA. Cancer and Thrombosis: Implications of Published Guidelines for Clinical Practice. Ann Oncol (2009) 20:1619-30. doi: 10.1093/annonc/ mdp068

4. Sussman TA, Li H, Hobbs B, Funchain P, McCrae KR, Khorana AA. Incidence of Thromboembolism in Patients With Melanoma on Immune Checkpoint Inhibitor Therapy and Its Adverse Association With Survival. J Immunother Cancer (2021) 9(1):e001719. doi: 10.1136/jitc-2020-001719

5. Moik F, Chan WE, Wiedemann S, Hoeller C, Tuchmann F, Aretin MB, et al. Incidence, Risk Factors, and Outcomes of Venous and Arterial Thromboembolism in Immune Checkpoint Inhibitor Therapy. Blood (2021) 137:1669-78. doi: 10.1182/blood.2020007878

6. Franco AT, Corken A, Ware J. Platelets at the Interface of Thrombosis, Inflammation, and Cancer. Blood (2015) 126:582-8. doi: 10.1182/blood-201408-531582

7. Margraf A, Zarbock A. Platelets in Inflammation and Resolution. J Immunol (2019) 203:2357-67. doi: 10.4049/jimmunol.1900899

8. Koupenova M, Clancy L, Corkrey HA, Freedman JE. Circulating Platelets as Mediators of Immunity, Inflammation, and Thrombosis. Circ Res (2018) 122:337-51. doi: 10.1161/CIRCRESAHA.117.310795

9. Ribeiro LS, Migliari Branco L, Franklin BS. Regulation of Innate Immune Responses by Platelets. Front Immunol (2019) 10:1320. doi: 10.3389/ fimmu.2019.01320

10. Ramadori P, Klag T, Malek NP, Heikenwalder M. Platelets in Chronic Liver Disease, From Bench to Bedside. JHEP Rep (2019) 1:448-59. doi: 10.1016/ j.jhepr.2019.10.001

11. Malehmir M, Pfister D, Gallage S, Szydlowska M, Inverso D, Kotsiliti E, et al. Platelet GPIbalpha Is a Mediator and Potential Interventional Target for NASH and Subsequent Liver Cancer. Nat Med (2019) 25:641-55. doi: $10.1055 /$ s-0038-1677172

12. Posch F, Thaler J, Zlabinger GJ, Konigsbrugge O, Koder S, Zielinski C, et al. Soluble Vascular Endothelial Growth Factor (sVEGF) and the Risk of Venous Thromboembolism in Patients With Cancer: Results From the Vienna Cancer and Thrombosis Study (CATS). Clin Cancer Res (2016) 22:200-6. doi: 10.1158/1078-0432.CCR-14-3358

\section{ACKNOWLEDGMENTS}

The authors wish to thank to all the authors who have contributed to this research topic and the reviewers for their fruitful comments. The authors thank Birgit Schneider for help with the preparation of artwork. This work was supported by grants from the Deutsche Forschungsgemeinschaft (German Research Council), Germany (GRK2099 "Hallmarks of Skin Cancer") and the Erich und Gertrud RoggenbuckStiftung, Germany.

13. Ay C, Simanek R, Vormittag R, Dunkler D, Alguel G, Koder S, et al. High Plasma Levels of Soluble P-Selectin Are Predictive of Venous Thromboembolism in Cancer Patients: Results From the Vienna Cancer and Thrombosis Study (CATS). Blood (2008) 112:2703-8. doi: 10.1182/ blood-2008-02-142422

14. Bauer AT, Suckau J, Frank K, Desch A, Goertz L, Wagner AH, et al. Von Willebrand Factor Fibers Promote Cancer-Associated Platelet Aggregation in Malignant Melanoma of Mice and Humans. Blood (2015) 125:3153-63. doi: 10.1182/blood-2014-08-595686

15. Briquet-Laugier V, Lavenu-Bombled C, Schmitt A, Leboeuf M, Uzan G, Dubart-Kupperschmitt A, et al. Probing Platelet Factor 4 Alpha-Granule Targeting. J Thromb Haemost (2004) 2:2231-40. doi: 10.1111/j.15387836.2004.01037.x

16. Lucotti S, Muschel RJ. Platelets and Metastasis: New Implications of an Old Interplay. Front Oncol (2020) 10:1350. doi: 10.3389/fonc.2020.01350

17. Gebremeskel S, LeVatte T, Liwski RS, Johnston B, Bezuhly M. The Reversible P2Y12 Inhibitor Ticagrelor Inhibits Metastasis and Improves Survival in Mouse Models of Cancer. Int J Cancer (2015) 136:234-40. doi: 10.1002/ ijc. 28947

18. Wojtukiewicz MZ, Hempel D, Sierko E, Tucker SC, Honn KV. Antiplatelet Agents for Cancer Treatment: A Real Perspective or Just an Echo From the Past? Cancer Metastasis Rev (2017) 36:305-29. doi: 10.1007/s10555-017-9683-Z

19. Rachidi S, Metelli A, Riesenberg B, Wu BX, Nelson MH, Wallace C, et al. Platelets Subvert T Cell Immunity Against Cancer via GARP-TGFbeta Axis. Sci Immunol (2017) 2(11):eaai7911. doi: 10.1126/sciimmunol.aai7911

Conflict of Interest: The authors declare that the research was conducted in the absence of any commercial or financial relationships that could be construed as a potential conflict of interest.

Publisher's Note: All claims expressed in this article are solely those of the authors and do not necessarily represent those of their affiliated organizations, or those of the publisher, the editors and the reviewers. Any product that may be evaluated in this article, or claim that may be made by its manufacturer, is not guaranteed or endorsed by the publisher.

Copyright (c) 2021 Schlesinger, Gobec and Bauer. This is an open-access article distributed under the terms of the Creative Commons Attribution License (CC BY). The use, distribution or reproduction in other forums is permitted, provided the original author(s) and the copyright owner(s) are credited and that the original publication in this journal is cited, in accordance with accepted academic practice. No use, distribution or reproduction is permitted which does not comply with these terms. 